\title{
Animal Models for Hepatic Fibrosis and Cirrhosis
}

\author{
Sherine A Mohammed and Eman E Abu-Deif* \\ Department of Histology, Faculty of Medicine, Sohag University, Egypt \\ *Corresponding author: Eman E Abu Deif, Department of Histology, Faculty of \\ Medicine, Sohag University, Egypt, Email: eman_elmadany@yahoo.com
}

\section{Editorial}

Volume 2 Issue 1

Received Date: November 19, 2018

Published Date: November 30, 2018
Abbreviations: NASH: Non-Alcoholic Steato-Hepatitis; HSC: Hepatic Stellate Cell; CBDL: Common Duct Ligation; NAFLD: Non Alcoholic Fatty Liver Disease; AFLD: Alcoholic Fatty Liver Disease.

\section{Introduction}

Liver cirrhosis represents a major health problem. No effective anti-fibrotic therapy is available up till now. Understanding the pathogenesis of liver fibrosis is essential for developing new strategies for anti- fibrotic therapies. Animal models may be used for this purpose $[1,2]$. Animal models have several advantages compared to clinical studies including; the possibility of taking samples at different time-points, the ability to control variables that cannot be controlled in humans, and the ability to study the implication of specific genes by using genetically modified animals [3]. However, in some liver pathologies associated with systemic diseases like nonalcoholic steato-hepatitis (NASH) there is no animal model can simulate the complex hepatic and extra-hepatic features [4]. Some differences exist between humans and animals. Viruses like hepatitis C or B do not infect rodent hepatocytes [5]. Alcoholic liver disease is difficult to be induced in rodents and rarely cause fibrosis [6].

When using animal models for studying liver cirrhosis multiple points must be considered including; reproducibility, specificity, feasibility, eliminating individual variations by using optimal number of animals and largely sampling the liver to avoid sampling error. Moreover, the different animal species and strains are different in fibrosis reproducibility due to different genetic susceptibility of different strains [3]. Liver Fibrosis is a wound-healing response to liver injury of various origins. It is defined by excessive deposition of extracellular matrix. Hepatic stellate cell (HSC) is the key fibrogenic cell. Fibrosis occurs on top of inflammation and necrosis which can be caused by many factors such as infection and intoxication [2]. The inflammatory reaction leads to activation of Kupffer cells which in turn stimulate hepatocyte regeneration via secretion of proliferative cytokines and activate HSC via secretion of fibrogenic cytokines. When the regenerative power of hepatocytes becomes exhausted, the degenerated hepatocytes are replaced with fibrous tissue produced by HSC [7].

\section{Types of Experimentally Induced Cirrhosis}

Several animal models of cirrhosis have been used. Animal models of liver cirrhosis can be induced by one of the following approaches:

\section{Hepatotoxin Induced Cirrhosis}

The most widely used hepatotoxic drugs-induced cirrhosis models are CCl4-induced cirrhosis, TTA-induced cirrhosis, and DMN-induced cirrhosis. These toxins are mainly metabolized by centri-lobular hepatocytes and cause centri-lobular liver damage [8]. As fibrosis extends, portal-central septa are formed (bridging fibrosis), and finally cirrhotic nodules are completely surrounded by fibrous septa. In humans, fibrosis is more frequently seen in peri-portal areas then the complete cirrhotic picture is formed. Many parameters should be considered when using hepato-toxins for induction of cirrhosis including; dosage, frequency, duration, dilution, and nature of the vehicle .In addition, the susceptibility of a given animal strain, depending on immunologic background, affects efficiency and severity of liver fibrosis development. Variation in the severity of fibrosis after $\mathrm{CCl} 4$ administration was observed in Balbc and C57BL/6 mice. TAA exposure leads to cirrhosis in a duration between 12 and 16 weeks in rats and between 16 and 24 weeks in mice [2]. 
TAA models have good survival rates, but less reproducibility, inflammatory cells were observed more than fibrous tissue in their livers [9]. DMN models are the worst of them because of their high mortality rates. In addition, their livers exhibited marked necrosis with only little fibrosis. DMN is also used to induce hepatocellular carcinoma in the context of chronic fibrosis [10].

The CCl4-induced model is a widely used model of hepatic cirrhosis .Repeated doses of CCl4 lead to HSC activation and development of progressive hepatic fibrosis. CCl4 models are considered to have the most acceptable survival rates and reproducibility [1]. But, CCl4-induced liver fibrosis reverses in a short time in contrast to TAA-induced fibrosis which persists for more than 2 months after TAA withdrawal. Multiple protocols for $\mathrm{CCl} 4$ administration are described (intra-peritoneal and subcutaneous injection, oral gavage, and inhalation) [2]. Subcutaneous injection has high survival rate with minimal adhesions [11].

\section{Biliary Cirrhosis}

Cholestatic liver diseases lead to ductular proliferation with periportal inflammation and then portal fibrosis. In animal models, this type of liver fibrosis can be induced by common duct ligation (CBDL) which leads to obstruction of bile flow and cholestasis. Liver cirrhosis could be established in 28 days after CBDL. The disadvantage of this model is the surgical complications which are more common in small animals like mice [8]. Other models of biliary cirrhosis were introduced to simulate primary sclerosing cholangitis; an auto immune disease leads to cholestasis. In these models cholangitis is induced chemically or by infectious agents. However, no perfect animal model resembling all features of primary sclerosing cholangitis is available Pollheimer MJ, et al. [12].

Diet-induced cholestatic liver injury as DDC-modified diet (0.1\% 3,5-diethoxycarbonyl-1,4- dihydrocollidine) is another type of biliary fibrosis models in which portal septa develop within 4-7 weeks [13].

\section{Immune Reaction-Induced Cirrhosis}

The antigen antibody complex deposition induces allergic and inflammatory reaction and then fibrosis develops. Prolonged administration of heterologous serum as porcine serum can induce cirrhosis in rodent models. Autoimmune hepatitis is a progressive T- cell mediated necro-inflammatory liver disease [14].
Schistosoma infection is a type of immunologically induced fibrosis [15].

\section{Genetic Modifications for Developing Cirrhosis}

Induced overexpression of certain pro-fibrotic genes and/or silencing of anti-fibrotic genes have been shown to induce cirrhosis in animals. The most important gene expression associated with hepatic fibrosis are platelet derived growth factor-beta [16] and transforming growth factor-beta $[17,18]$. Example for gene silencing or gene knockout is mdr2 gene silencing for sclerosing cholangitis with subsequent fibrosis [19].

\section{Non Alcoholic Fatty Liver Disease(NAFLD)}

Steato-hepatitis models can be induced by hypercaloric diet and methionine or choline deficient diet. In these models, hepatocellular degeneration in the form of ballooning and steatosis followed by liver cell injury that elicit inflammatory reaction, steato-hepatitis with subsequent fibrosis and cirrhosis. In the models induced by choline deficient diet moderate peri-cellular fibrosis with only porto-central bridges are present $[4,20]$.

\section{Alcoholic Fatty Liver Disease(AFLD)}

Alcoholic liver disease is the first cause of liver cirrhosis in Europe. But in animal models, no significant fibrosis develops with alcohol administration only hepatocyte steatosis. This is because rodents rapidly metabolize alcohol. So, it is difficult to maintain high blood level of alcohol in rodents as in human. Only the Intragastric feeding model of Tsukamoto-French could develop fibrosis by sustained alcohol administration via a gastric cannula [6].

\section{Mice with Humanized Liver}

These are immune deficient mice whose livers are destructed and engrafted with human hepatocytes that proliferate and restore a human liver inside the mouse. These are promising models for studying many drug metabolism and allow the mice to be infected with viruses with subsequent fibrosis [5,21-24].

\section{References}

1. Fortea JI, Fernández Mena C, Puerto M, Ripoll C, Almagro J, et al. (2018) Comparison of Two Protocols of Carbon Tetrachloride-Induced Cirrhosis in RatsImproving Yield and Reproducibility. Scientific Reports 8(1): 9163. 
2. Liedtke C, Luedde T, Sauerbruch T, Scholten D, Streetz K, et al. (2013) Experimental Liver fibrosis research: update on animal models, legal issues and translational aspects. Fibrogenesis \& Tissue Repair 6(1): 19.

3. Delire B, Starkel P, Leclercq I (2015) Animal Models for Fibrotic Liver Diseases: What We Have, What We Need, and What Is under Development. J Clin Transl Hepatol 3(1): 53-66.

4. Farrell GC, Larter CZ (2006) Nonalcoholic fatty liver disease: from steatosis to cirrhosis. Hepatology 43(2): S99-S112.

5. Washburn ML, Bility MT, Zhang L, Kovalev GI, Buntzman A, et al. (2011) A humanized mouse model to study hepatitis $\mathrm{C}$ virus infection, immune response, and liver disease. Gastroenterology 140(4): 13341344

6. Gao B, Xu MJ, Bertola A, Wang H, Zhou Z, et al. (2017) Animal Models of Alcoholic Liver Disease: Pathogenesis and Clinical Relevance. Gene expression 17(3): 173-186.

7. Xu T, Ni MM, Xing L, Li XF, Meng XM, et al. (2016) NLRC5 regulates TGF-beta1-induced proliferation and activation of hepatic stellate cells during hepatic fibrosis. Int J Biochem Cell Biol 70: 92-104.

8. Vanheule E, Geerts AM, Van Huysse J, Schelfhout D, Praet M, et al. (2008) An intravital microscopic study of the hepatic microcirculation in cirrhotic mice models: relationship between fibrosis and angiogenesis. Int J Exp Pathol 89(6): 419-432.

9. Nascimento M, Piran R, Da Costa RM, Giordani, MA, Carneiro FS (2018) Hepatic injury induced by thioacetamide causes aortic endothelial dysfunction by a cyclooxygenase-dependent mechanism. Life Sci 212: 168-175.

10. Cheng Y, Mai JY, Wang MF, Chen GF, Ping J (2017) Antifibrotic effect of total flavonoids of Astmgali Radix on dimethy Initrosamine-induced liver cirrhosis in rats. Chin J Integr Med 23(1): 48-54.

11. Abu-Dief E, Mohammed D, Abd El-Haliem N, Mohammed S, El-Badry AM (2018) Impact of omega-3 fatty acids on evolution of carbon tetrachloride(CCl4) induced liver cirrhosis in mice: A histological and immunohistochemical study. Egyptian Journal of Histology 41(1): 61-72.
12. Pollheimer MJ, Fickert P (2015) Animal models in primary biliary cirrhosis and primary sclerosing cholangitis. Clin Rev Allergy Immunol 48(2-3): 207217.

13. Fickert $P$, Stöger U, Fuchsbichler A, Moustafa $T$, Marschall HU, et al. (2007) A new xenobiotic-induced mouse model of sclerosing cholangitis and biliary fibrosis. The American journal of pathology 171(2): 525-536.

14. Christen U (2018) Animal models of autoimmune hepatitis. Biochim Biophys Acta Mol Basis Dis.

15. Huang YF, Zhang SL, Jin F, Cheng, D, Zhou et al. (2013) Activity of corilagin on post-parasiticide liver fibrosis in Schistosomiasis animal model. Int J Immunopathol Pharmacol 26(1): 85-92.

16. Czochra P, Klopcic B, Meyer E, Herkel J, Garcia-Lazaro $\mathrm{JF}$, et al. (2006) Liver fibrosis induced by hepatic overexpression of PDGF-B in transgenic mice. J Hepatol 45(3): 419-428.

17. Clouthier DE, Comerford SA, Hammer RE (1997) Hepatic fibrosis, glomerulosclerosis, and a lipodystrophy-like syndrome in PEPCK-TGF-beta1 transgenic mice. J Clin Invest 100(11): 2697-2713.

18. Dropmann A, Dediulia T, Breitkopf Heinlein K, Korhonen H, Janicot M, et al. (2016) TGF-beta1 and TGF-beta2 abundance in liver diseases of mice and men. Oncotarget 7(15): 19499-19518.

19. Hall C, Ehrlich, L, Meng F, Invernizzi, P, Bernuzzi F, et al. (2017) Inhibition of micro RNA-24 increases liver fibrosis by enhanced menin expression in Mdr2(-/-) mice. J Surg Res 217: 160-169.

20. Wobser H, Dorn C, Weiss TS, Amann T, Bollheimer C, et al. (2009) Lipid accumulation in hepatocytes induces fibrogenic activation of hepatic stellate cells. Cell Res 19(8): 996-1005.

21. Shultz, LD, Ishikawa F, Greiner DL (2007) Humanized mice in translational biomedical research. Nat Rev Immunol 7(2): 118-130.

22. Theocharides AP, Rongvaux A, Fritsch K, Flavell RA, Manz MG (2016) Humanized hemato-lymphoid system mice. Haematologica 101(1): 5-19. 
23. Walsh NC, Kenney LL, Jangalwe S, Aryee KE, Greiner DL, et al. (2017) Humanized Mouse Models of Clinical Disease. Annual review of pathology 12: 187-215.
24. Guo J, Li Y, Shan Y, Shu C, Wang F, et al. (2018) Humanized mice reveal an essential role for human hepatocytes in the development of the liver immune system. Cell Death \& Disease 9(6): 667. 